\title{
Discovery of prognostic biomarkers for predicting lung cancer metastasis using microarray and survival data
}

\author{
Hui-Ling Huang ${ }^{1,2^{*}}$, Yu-Chung Wu ${ }^{3}$, Li-Jen Su${ }^{4}$, Yun-Ju Huang ${ }^{5}$, Phasit Charoenkwan', Wen-Liang Chen², \\ Hua-Chin Lee ${ }^{1,2}$, William Cheng-Chung $\mathrm{Chu}^{6}$ and Shinn-Ying $\mathrm{Ho}^{1,2^{*}}$
}

\begin{abstract}
Background: Few studies have investigated prognostic biomarkers of distant metastases of lung cancer. One of the central difficulties in identifying biomarkers from microarray data is the availability of only a small number of samples, which results overtraining. Recently obtained evidence reveals that epithelial-mesenchymal transition (EMT) of tumor cells causes metastasis, which is detrimental to patients' survival.

Results: This work proposes a novel optimization approach to discovering EMT-related prognostic biomarkers to predict the distant metastasis of lung cancer using both microarray and survival data. This weighted objective function maximizes both the accuracy of prediction of distant metastasis and the area between the disease-free survival curves of the non-distant and distant metastases. Seventy-eight patients with lung cancer and a follow-up time of 120 months are used to identify a set of gene markers and an independent cohort of 26 patients is used to evaluate the identified biomarkers. The medical records of the 78 patients show a significant difference between the disease-free survival times of the 37 non-distant- and the 41 distant-metastasis patients. The experimental results thus obtained are as follows. 1) The use of disease-free survival curves can compensate for the shortcoming of insufficient samples and greatly increase the test accuracy by $11.10 \%$; and 2) the support vector machine with a set of 17 transcripts, such as CCL16 and CDKN2AIP, can yield a leave-one-out cross-validation accuracy of 93.59\%, a test accuracy of $76.92 \%$, a large disease-free survival area of $74.81 \%$, and a mean survival prediction error of 3.99 months. The identified putative biomarkers are examined using related studies and signaling pathways to reveal the potential effectiveness of the biomarkers in prospective confirmatory studies.
\end{abstract}

Conclusions: The proposed new optimization approach to identifying prognostic biomarkers by combining multiple sources of data (microarray and survival) can facilitate the accurate selection of biomarkers that are most relevant to the disease while solving the problem of insufficient samples.

Keywords: Distant metastasis, Genetic algorithm, Lung cancer, Microarray, Prognostic biomarker, Survival curve

\section{Background}

Primary lung cancer is very heterogeneous in its clinical presentation, histopathology, and treatment response [1]. Differentiating between an occurrence of a new primary lung cancer and a recurrence of lung cancer is often difficult. Conventionally, lung cancers have been divided

\footnotetext{
*Correspondence: hlhuang@mail.nctu.edu.tw; syho@mail.nctu.edu.tw ${ }^{1}$ Institute of Bioinformatics and Systems Biology, National Chiao Tung University, Hsinchu, Taiwan

${ }^{2}$ Department of Biological Science and Technology, National Chiao Tung University, Hsinchu, Taiwan

Full list of author information is available at the end of the article
}

into non-small-cell lung cancer (NSCLC) and small-cell lung cancer (SCLC). The stage of each cancer is the most significant predictor of survival. Cancer metastasis and the emergence of drug resistance are the major causes of the failure of treatment for lung cancer. Thus, therapy for lung cancer that takes into account distant metastasis and drug resistance is an emerging field of research. Prognostic biomarkers are expected to be useful in predicting the probable course of lung cancer metastases, and they importantly affect the aggressiveness of therapy. Some new promising strategies for biomarker 
discovery include microarray-based profiling at the DNA and mRNA levels, and mass-spectrometry-based profiling at the protein and peptide levels [2]. The combination of multiple biomarkers is generally agreed to increase diagnostic sensitivity and specificity over the use of individual markers.

During cancer progression, some tumor cells acquire new characteristics, such as over-expression of epithelialmesenchymal transition (EMT) markers, and undergo profound morphogenetic changes. EMT is a process in which epithelial cells lose their cell polarity and cell-cell adhesion, and gain migratory and invasive properties, becoming mesenchymal cells $[3,4]$. EMT plays an important role in cancer progression and provides a new basis for understanding the progression of carcinoma towards dedifferentiated and more malignant states [3,4]. Additionally, EMT affects cancer cell invasion, resistance to apoptosis, and stem cell features [5]. Growth factors [6,7], liganddependent nuclear receptors [8], transcription regulators $[3,9]$, cytokine $[7,10]$, and kinase $[11,12]$, which are potential regulators that are related to EMT have been identified in the literature. Signaling pathways that are activated by intrinsic or extrinsic stimulation converge on the transcriptional factors and regulate phenotypic changes of cancer cells [9].

DNA microarrays perform the simultaneous interrogation of thousands of genes and provide an opportunity to measure a tumor from multiple perspectives. Microarray-based techniques generally provide detailed observations at gene activities in tumors and generate opportunities for finding therapeutic targets. As a high-throughput technology at the molecular level, DNA microarray-based methods have clear advantages over traditional histological examinations and have been extensively used in cancer research to predict more accurately clinical outcomes and potentially improve patient management. Studies indicate that microarray techniques greatly facilitate accurate tumor classification and predicted outcome in terms of, for example, tumor stage, metastatic status, and patient survival, offering some hope for personalized medicine [13-15].

Methods for predicting lung cancer metastasis involve feature (gene) selection and classifier design. Feature selection identifies a subset of differentially-expressed genes that are potentially relevant to distinguishing different classes of samples [16]. One of the central difficulties in investigating microarray classification and gene selection is the availability of only a small number of samples, compared to the large number of genes in a sample [16]. Hierarchical clustering [17] is one of the most commonly used approaches in microarray studies. However, hierarchical clustering (or any purely correlative technique) cannot alone provide a rational biological basis for disease classification [18]. Generally, univariate analysis is conducted to reduce feature size and, then, a support vector machine (SVM) [19] or maximum likelihood classification [20] with an effective feature selection method is used to identify a small set of informative genes. The most challenging task is to avoid overfitting a small number of samples, resulting in the poor performance of independent tests.

In this work, the medical records of 78 patients with lung cancer and a follow-up time of 120 months reveal significant difference between the disease-free survival times of the 37 non-distant- and the 41 distantmetastasis patients. To solve the problem of insufficient samples, this work proposes a novel optimization approach to discovering EMT-related prognostic biomarkers for predicting the distant metastasis of lung cancer using both microarray and survival data. The proposed optimal gene selection method incorporates gene expression profiles and their corresponding diseasefree survival curves of patients to design a fitness function for using an intelligent genetic algorithm [21]. The set of 78 samples is used to identify a set of gene markers and an independent cohort of 26 samples is used to evaluate the identified biomarkers. The experimental results show that disease-free survival curves can compensate for the insufficient samples and the SVM with a set of 17 transcripts can yield high prediction accuracies of distant metastasis and disease-free survival time. The putative biomarkers for predicting the distant metastasis of lung cancer are examined using relevant signaling pathways to reveal the potential of biomarkers.

\section{Methods}

\section{Data sets}

\section{RNA isolation and microarray platform}

Illumina Sentrix-6 Whole-Genome Expression BeadChips are relatively new microarray platforms, that have been used in many microarray studies in the past few years [22]. Physically, each Sentrix-6 BeadChip consists of 12 equally-spaced strips of beads. Each pair of adjacent strips comprises a single microarray and is hybridized with a single RNA sample [22]. The used microarray is Illumina of HumanWG-6 BeadChip Kit Support. Fresh-frozen specimens were removed from liquid nitrogen and homogenized using TissuLyzer in RLT buffer of RNeasy isolation kit, both from Qiagen. Total RNA was extracted from fresh-frozen tumors followed the manufacturer's suggestion, purified by RNeasy mini kit, and checked by NanoDrop spectrophotometer and Agilent Bioanalyzer for quantity and quality. Biotin labeled cRNA was prepared from Illumina TotalPrepTM RNA amplification kit, Life Technologies. One and half ug cRNA was hybridized to the Illumina Multi-sample Human WG-6 v3.0 chip according to manufacturer's instructions. Globe normalization was used to normalize for signal intensity of chips. This microarray of a sample has 48,803 transcripts. 


\section{Cohorts of lung cancer}

The dataset of 78 lung cancer samples, comprising 37 non-distant- and 41 distant-metastasis samples as well as their corresponding disease-free survival time, comes from Taipei Veterans General Hospital (TVGH) in Taiwan. This work developed prognostic models using these 78 samples. To evaluate the potential of putative biomarkers and the gene-discovery method for identifying a small set of genes that can be used to predict distant metastasis of lung cancer, a cohort of 26 samples, comprising 6 non-distant- and 20 distant-metastasis patients of TVGH, is utilized as an independent test dataset. This study as well as the tissue procurement protocol were approved by the Institutional Review Board of TVGH (VGHIRB No. 2013-04-015 AC), and written informed consent was obtained from all patients. The datasets of 78 and 26 samples consisting of microarray data and disease-free survival time that the patient identifiers have been removed are given in Additional file 1: Table S1 and Additional file 2: Table S2, respectively.

\section{Characteristics of cohorts}

Patient survival is a major clinical parameter that is used to evaluate the efficacy of a particular therapy. Diseasefree survival, used herein, is defined as the time between surgery and the occurrence of an event (death or distant metastasis). The censored data are that when the event did not occur, and the survival time is that between surgery and the last follow-up date. Figure 1 shows statistics concerning disease-free survival times for 78 lung cancer patients with a follow-up time of 120 months. The mean times of disease-free survival for non-distant and distant metastasis are 73.08 and 14.02 months, respectively. A $t$-test with $\mathrm{p}$-value $=7.99 \mathrm{E}-22$ reveals a significant difference $(p<0.001)$ between the disease-free survival times of the 37 non-distant- and the 41 distantmetastasis patients. The result suggests that distant metastasis is strongly correlated to patients' survival. Some characteristics of these 78 patients were summarized in Table 1. From the results of Fisher's exact test, there was no significant association between distant metastasis and the interested factors such as age, sex, smoking, tumor size, $\mathrm{pN}$ and $\mathrm{pM}$ status, histologic type, and differentiation ( $p>0.05)$. Notably, there was a strong association between distant metastasis and pathologic stage $(\mathrm{p}=3.0 \mathrm{E}-5)$.

\section{EMT-related transcripts}

Ingenuity Knowledge Base (IKB; http://www.ingenuity. com) is a repository of biological interactions and functional annotations that are created from millions of individually modeled relationships among proteins, genes, complexes, cells, tissues, metabolites, drugs, and diseases. IKB provides comprehensive, species-specific knowledge about the function and regulation of genes, tissue and cell line expression patterns, clinical biomarkers, subcellular locations, mutations, and disease associations. First, EMT-related genes annotated as "growth factor", "ligand-dependent nuclear receptor", "transcription regulator", "cytokine", or "kinase" in the IKB are identified. As a result, 4,314 transcripts are identified in the HumanWG-6 microarray data, comprising 233 transcripts of growth factor, 103 transcripts of ligand-dependent nuclear receptor, 2,465 transcripts of transcription regulator, 276 transcripts of cytokine, and 1,237 transcripts of kinase. Second, univariate $t$-test analysis is used to calculate the $p$-values of individual transcripts between two classes. The genes with a very small $p$-value are useful in predicting distant metastasis. There are 474 top-ranked transcripts according to the p-value selected from the 4,314 EMTrelated transcripts. The training dataset of 78 samples with

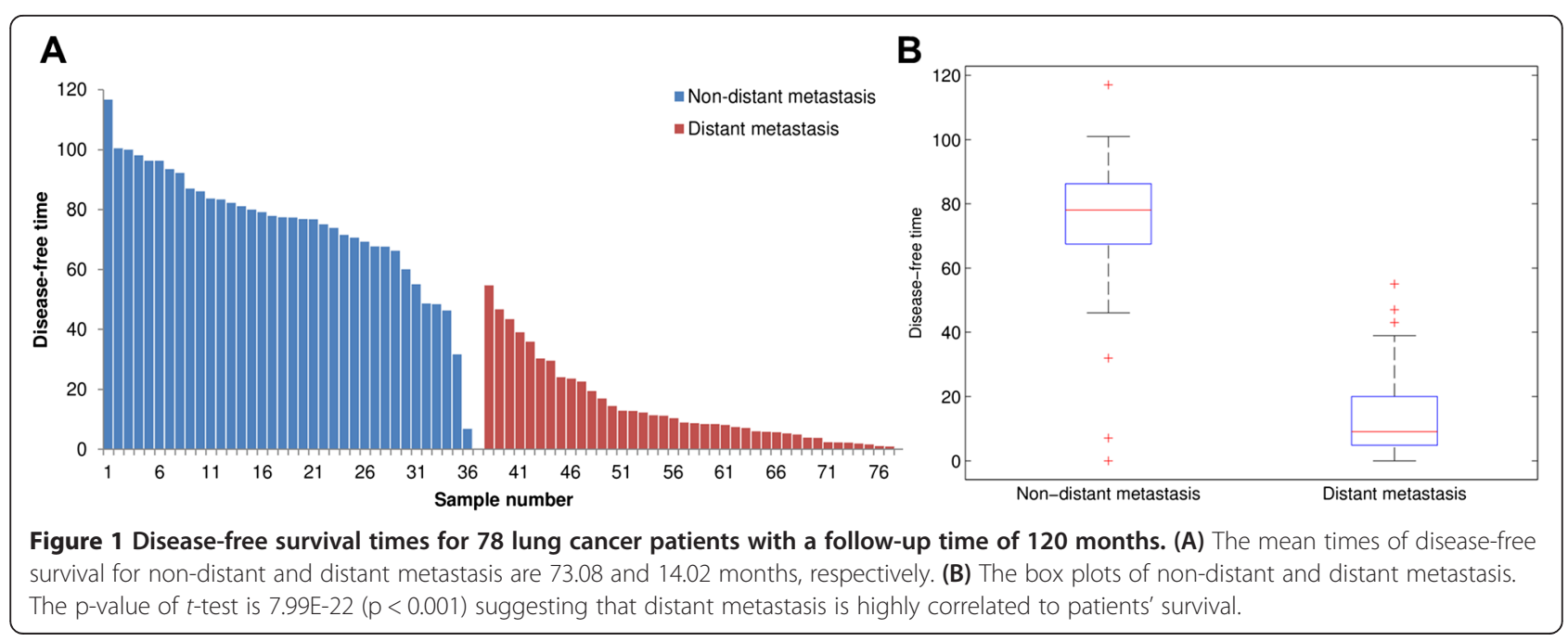


Table 1 Selected characteristics of participants according to NSCLC

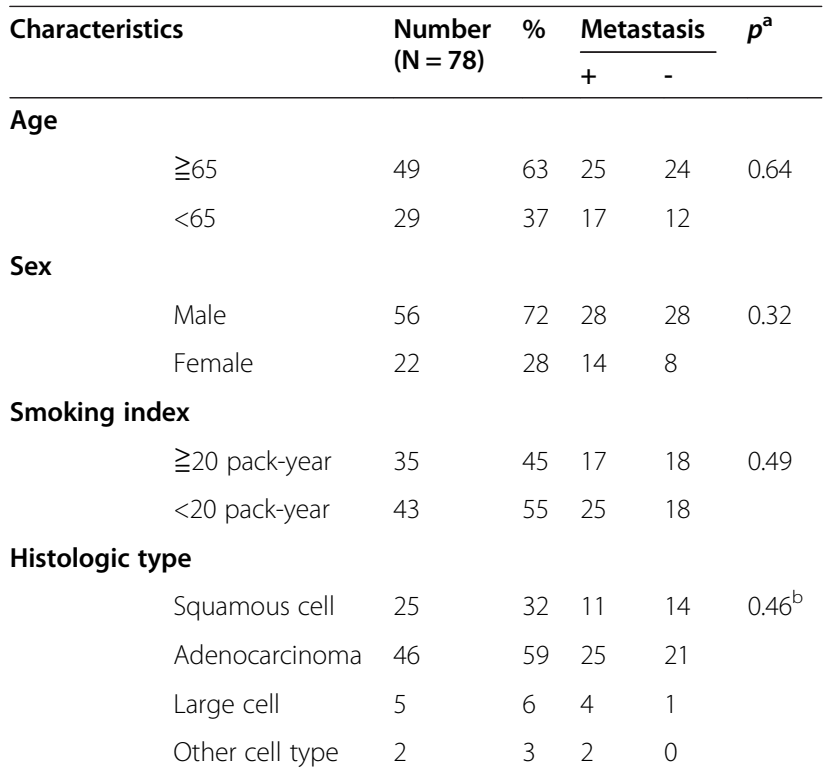

Differentiation

$\begin{array}{llllll}\text { Well } & 5 & 6 & 1 & 4 & \\ \text { Moderate } & 51 & 65 & 28 & 23 & 0.80^{\mathrm{b}} \\ \text { Poor } & 22 & 28 & 13 & 9 & \end{array}$

Tumor size

$\begin{array}{lllll}\geqq 5 \mathrm{~cm} & 18 & 23 & 11 & 7 \\ <5 \mathrm{~cm} & 59 & 76 & 31 & 28\end{array}$

pN status

$\begin{array}{llllll}\text { Positive } & 54 & 69 & 25 & 29 & 0.09 \\ \text { Negative } & 25 & 32 & 17 & 7 & \end{array}$

pM status

$\begin{array}{llllll}\text { Positive } & 5 & 6 & 5 & 0 & 0.20 \\ \text { Negative } & 73 & 94 & 37 & 36 & \end{array}$

Pathologic stage

\begin{tabular}{llllll} 
Stage I & 36 & 46 & 10 & 26 & $3.0 \mathrm{E}-5^{c}$ \\
Stage II & 11 & 14 & 7 & 4 & \\
Stage III & 26 & 33 & 20 & 6 & \\
Stage IV & 5 & 6 & 5 & 0 & \\
\hline
\end{tabular}

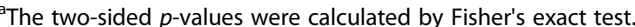

${ }^{\mathrm{b}}$ The $p$-values were calculated using the two variables with the largest numbers of patients.

'The $p$-value was calculated to measure the association between two variables (Stage I and Stages II, III and IV).

the 474 EMT-related transcripts is used to identify a small set of prognostic biomarkers that is predictive of the distant metastasis of lung cancer.

\section{Methods}

\section{Disease-free survival area}

The Kaplan-Meier survival curves reveal a significant difference between the survival times of patients in the two classes. The disease-free survival area is calculated between the two survival curves from zero to the maximum disease-free survival time (117 months in this work). The disease-free survival area is represented as a percentage, which is the ratio of this area to the maximum area. The gene set with a large disease-free survival area is expected to have a strong ability to distinguish a non-distant metastasis from a distant metastasis of lung cancer.

\section{Fitness function}

Microarray data contain valuable information about a huge gene set but often suffer from a small number of available samples. In the design of classifiers with gene selection, there exist numerous candidate sets of genes that can achieve high training accuracies for predicting distant metastasis. However, most of the candidate gene sets have relatively low accuracies of independent tests that result in overtraining. The right sets of biomarkers should have high prediction accuracies for both training and test datasets. To cope with the overtraining problem, it is essential to identify the right sets of biomarkers by discarding the gene sets of overtraining. Recently obtained evidence reveals that distant metastasis from lung cancer is detrimental to patients' survival [23]. Moreover, Figure 1 suggests that distant metastasis is strongly related to patients' survival. Thus, it is hypothesized that the right set of biomarkers can also be used to effectively predict patients' survival. This work proposes a hybrid approach that uses two kinds of resources, microarray data and the disease-free survival data, to identify a set of biomarkers.

The fitness function provides the only means by which genetic algorithms (GAs) optimize all system parameters that are encoded in a GA-chromosome. The three objectives of designing the predictor of lung cancer metastasis and discovering a set of genes using GA-based optimization methods are as follows. The first objective is to maximize the classification accuracy (denoted as $A c c$ ) of the SVM classifier; the second is to maximize the disease-free survival area (denoted as Asurv), and the last is to identify a small set of informative genes. The values of Acc and Asurv are in the range [0, 1]. The two maximum objectives without conflicting each other can be combined into a weighted objective function $f(G)$ as follows.

$$
\operatorname{Max} f(G)=w \times A c c+(1-w) \times \operatorname{Asurv}
$$

where $w$ denotes a positive weight in the range $[0,1]$ which is determined according to the preferences for individual objectives, and $G$ denotes the selected gene set. Generally, maximizing $f(G)$ is the major objective and the number of selected genes is restricted within a 
relatively small range (as will be discussed in the next section). If $w=1.0$, then the fitness function degenerates to the conventional one that uses no clinical outcome (disease-free survival curve). The fitness function using the overall accuracy Acc is suitable for balanced datasets (almost equal populations of metastatic positive and negative samples). When applying to imbalanced datasets, random over-sampling methods such as SMOTE which is short for Synthetic Minority Over-sampling Technique [24] can be used to adjust these datasets to balanced datasets.

\section{Gene selection using IBCGA}

Selecting a minimal number of informative genes while maximizing the prediction performance of distant metastasis is a bi-objective $0 / 1$ combinatorial optimization problem. This work propose a novel method for identifying a small number $m$ of informative genes from a large number $n$ of candidate genes for prediction and biomarker discovery based on an inheritable bi-objective combinatorial genetic algorithm (IBCGA) [25] with SVM classifiers. IBCGA has been previously used to identify a small set of properties from 531 physicochemical properties for predicting the immunogenicity of MHC class I binding peptides [26]. Feature selection is a combinatorial optimization problem $\mathrm{C}(n, m)$ with a huge search space of size $C(n, m)=n ! /(m !(n-m) !))$. The IBCGA uses an intelligent genetic algorithm (IGA) [21] with an inheritance mechanism [25] to search efficiently for the solutions $S_{\mathrm{r}}$ to $\mathrm{C}(n, r)$ and $S_{\mathrm{r}+1}$ to $\mathrm{C}(n, r+1)$ by inheriting the good solution $S_{\mathrm{r}}$. IGA that is based on orthogonal experimental design uses a divide-and-conquer strategy and a systematic reasoning method rather than a conventional generate-and-go method to solve efficiently the large-scale combinatorial optimization problem. The SVM-based training model uses the prediction performance of leave-one-out cross-validation (LOOCV) as the fitness function in using IBCGA with the whole training set.

The input for the SVM-based model design procedure is a training dataset that is composed of two classes (distant and non-distant metastases). The output of the procedure includes a set of $m$ selected transcripts and an SVM classifier with associated parameter settings of $\gamma$ and $C$. A radial basis kernel function $\exp \left(-\gamma|| x_{\mathrm{i}}-x_{\mathrm{j}} \|^{2}\right)$ is adopted, where $x_{\mathrm{i}}$ and $x_{\mathrm{j}}$ are training samples, and $\gamma$ is a kernel parameter. In this work, $\gamma \in\left\{2^{-7}, 2^{-6}, \ldots, 2^{8}\right\}$ and $C \in\left\{2^{-7}, 2^{-6}, \ldots, 2^{8}\right\}$. Each sample is represented using an $n$-dimensional feature vector $\mathrm{P}=\left[p_{1}, p_{2}, \ldots, p_{\mathrm{n}}\right]$. In this work, $n=474$. The IGA-chromosome consists of $n$ binary IGA-genes $f_{\mathrm{i}}$ to select features and two 4-bit genes for encoding $\gamma$ and $C$. The corresponding feature $p_{\mathrm{i}}$ (the $i$-th transcript) is excluded from the SVM classifier if $f_{\mathrm{i}}=0$, and is included if $f_{i}=1$. Let $m$ be the sum of $f_{i}$.
The IBCGA with the fitness function $f(G)$ that uses LOOCV can simultaneously obtain a set of solutions, $S_{n}$ where $r=r_{\text {start }}, r_{\text {start }}+1, \ldots, r_{\text {end }}$ in a single run. In this work, the parameter settings are $r_{\text {start }}=10, r_{\text {end }}=30$, $N_{\text {pop }}=60, p_{c}=0.8, p_{m}=0.05$, and Gmax $=60$. The customized IBCGA for transcript selection is given below.

Step 1 (Initiation) Randomly generate an initial population of $N_{\text {pop }}$ individuals. All $n$ binary genes $f_{\mathrm{i}}$ have $r 1 \mathrm{~s}$ and $n-r 0 \mathrm{~s}$ where $r=r_{\text {start }}$.

Step 2 (Evaluation) Evaluate the fitness values of all individuals using $f(G)$.

Step 3 (Selection) Use a conventional method of tournament selection that selects the winner from two randomly selected individuals to generate a mating pool.

Step 4 (Crossover) Select $p_{c} \cdot N_{\text {pop }}$ parents from the mating pool to perform orthogonal array crossover [25] on selected pairs of parents where $p_{c}$ is the probability of crossover operations.

Step 5 (Mutation) Apply a conventional mutation operator to the randomly selected $p_{m} \cdot N_{\text {pop }}$ individuals (except the best individual) in the new population where $p_{m}$ is the probability of mutation operations.

Step 6 (Termination test) If the stopping condition (reaching Gmax generations) for obtaining the solutions $S_{r}$ is satisfied, then output the best individual as $S_{r}$. Otherwise, go to Step 2.

Step 7 (Inheritance) If $\mathrm{r}<r_{\text {end }}$, then randomly change one bit in the binary genes $f_{\mathrm{i}}$ for each individual from 0 to 1 ; increase $r$ by one, and go to Step 2 .

Step 8 (Non-deterministic) Perform Steps $1-7$ for $R$ (=30 in this work) independent runs and obtain the best of the $R$ solutions. The best solution can be determined by considering the most accurate one $\left(S_{a}\right)$ with the highest fitness value or the robust one $\left(S_{b}\right)$ with the highest score of appearance [27]. The appearance score considers both the fitness value as well as the mean number of times for individual genes selected in the $R$ runs.

Notably, all genetic algorithms search for globally optimal solutions but their outputs are non-deterministic because of randomization. Therefore, the common approach to solving with the non-deterministic problem is to perform a number of independent runs to evaluate the final answer. In this work, the answers obtained in all $R$ runs are utilized efficiently, as described in the next section.

\section{Gene selection using a sequential backward selection method}

An IGA-based gene selection method with SVM (known as ESVM) [19] can obtain a high prediction accuracy of $96.88 \%$ with a mean number of 10.0 to select genes from 11 benchmark datasets concerning various cancers using 10 -fold cross-validation (10-CV). For two two-class tumor datasets, ESVM yields a mean number of 4.65 
and a classification accuracy of $97.82 \%$. An IGA-based gene selection method uses a maximum likelihood (MLHD) classifier [20] to select a minimal number of relevant genes for accurate classification of tumor samples. The experimental results show that the hybrid method IGA/MLHD outperforms existing methods in terms of the number of selected genes (9.86 on average), classification accuracy (mean accuracy of 96.20\%), and robustness of the selected genes based on 11 human cancer-related gene expression datasets.

In this work, the IBCGA that is based on IGA with an SVM selects a small set of genes that are relevant to the distant metastasis of lung cancer while maximizing the fitness function. Since the number of samples (78) is very small, the IBCGA can identify a very small number $m$ of genes and obtain a very high training accuracy with $m<10$. However, a very small number of genes can provide a very high training accuracy (either LOOCV or $10-\mathrm{CV}$ ) but the independent accuracy is not always satisfactory owing to overtraining. Moreover, a feasible set of biomarkers for yielding the high tumor prediction accuracy on an independent test dataset often has more than 10 genes. Therefore, this work proposes a novel methodological approach to alleviating the overtraining problem in two ways: 1 ) by utilizing additional clinical data, i.e. the disease-free survival curve, and 2) using a sequential backward selection (SBS) method to select the best set of transcripts from all of the selected transcripts of the $R=30$ runs of the IBCGA. For each run, the IBCGA selects at least 10 transcripts $\left(r_{\text {start }}=10\right)$. Sequential backward selection starts from the full set of the 30 transcripts with the highest appearance times, and sequentially removes transcript $x$ from $G$ (called the G-x set) that results in the smallest decrease of the value of the objective function $f(G-x)$. Notably, the removal of a feature may actually increase the value of the objective function such that $f(G-\mathrm{x})>f(G)$.

\section{Results and discussion}

\section{Performance evaluation with various weights}

To determine the best value of the weight $w$ and prevent overtraining in the subsequent design of gene selection methods, all the 78 samples are randomly divided into five groups, of which four are used as a training set and the other serves as an independent test set. Each group of samples serves as a test set in turn. Four experiments with $w$ values of 1.0, 0.8, 0.5, and 0.2 are conducted. Table 2 shows the mean accuracies of the independent test for various weights. The results reveal that the best performance is achieved using $w=0.8$ with a test accuracy of $74.82 \%$. The conventional method has an accuracy of $61.62 \%$ when the disease-free survival area is not used $(w=1.0)$. The
Table 2 Accuracies of the independent test for various weights in the fitness function

\begin{tabular}{ll}
\hline Weight $w$ & Test accuracy (\%) \\
\hline 1.0 & 61.62 \\
0.8 & 74.82 \\
0.5 & 62.15 \\
0.2 & 51.69 \\
\hline
\end{tabular}

additional use of a survival curve can compensate for the shortcomings of insufficient microarray samples and improve the test accuracy $13.20 \%$.

\section{Identifying a gene set using IBCGA and sequential backward selection}

The IBCGA identifies a small number $m$ of transcripts from $n=474$ candidate transcripts while maximizing the fitness function $f(\mathrm{G})$. The results show that $m=10$ has a very high value of $f(\mathrm{G})$. The IBCGA with $R=30$ runs yields 30 sets of transcripts. The most accurate solution $S_{a}$ and the most robust solution $S_{b}$ are recorded. The number of appearances of each selected transcript in the 30 runs is recorded. Table 3 lists statistical results concerning the number of appearances for the 30 transcripts with the highest appearance frequency. The genes with rank 1 are $\mathrm{FBJ}$ murine osteosarcoma viral oncogene homolog B (FOSB) and microtubule associated serine/ threonine kinase 1 (MAST1), which were selected 12 times. The gene forkhead box E1 (FOXE1) has two transcripts with ID numbers 6250309 and 3450692, named FOXE1-1 and FOXE1-2, at ranks nine and 13, respectively. Similarly, the gene protein kinase $\mathrm{C}$ beta (PRKCB1) has two transcripts with ID numbers 3460564 and 5090563, named PRKCB1-1 and PRKCB1-2, at ranks 23 and 27, respectively. The set of 30 transcripts was used in the sequential backward selection (SBS) method for further identifying a set of prognostic biomarkers that are effective and stable in predicting lung cancer metastasis. Figure 2 shows the results of the SBS method for $w=0.8$ and 1.0. Table 4 shows the performance of the IBCGA and SBS methods for $w=0.8$ and 1.0.

From Figure 2, the SBS method with 17 transcripts and $w=0.8$ has the largest disease-free survival area $($ Asurv $=74.81 \%)$ and prediction accuracy $(A c c=93.59 \%)$. The largest Asurv is $70.92 \%$ and $A c c=91.03 \%$ when a set of 15 transcripts was adopted for $w=1.0$. From Table 4 , the $S_{a}$ solution has a set of 10 transcripts and is associated with a performance of $A c c=95.95 \%$ and Asurv $=$ $70.01 \%$ for $w=0.8$. The $S_{b}$ solution has a set of 10 transcripts and is associated with a performance of $A c c=$ 92.02\% and Asurv $=63.81 \%$ for $w=0.8$. Consider that 1 ) the 30 transcripts with high frequency are selected; 2) the test accuracy of $w=0.8$ is higher than that of $w=1.0$; and 3) the set of 17 transcripts yields the largest disease- 
Table 3 The 30 top-ranked transcripts in terms of selected times from 30 runs

\begin{tabular}{llllll}
\hline Rank & Transcript & Frequency & Rank & Transcript & Frequency \\
\hline 1 & FOSB & 12 & 16 & CDKL5 & 3 \\
2 & MAST1 & 12 & 17 & DCK & 3 \\
3 & CCL15 & 8 & 18 & KLF12 & 3 \\
4 & MAK & 6 & 19 & ZAP70 & 3 \\
5 & SF1 & 5 & 20 & BACH2 & 3 \\
6 & HDAC9 & 5 & 21 & YSK4 & 3 \\
7 & YSK4 & 4 & 22 & ELF5 & 3 \\
8 & EDN1 & 4 & 23 & PRKCB1-1 & 2 \\
9 & FOXE1-1 & 4 & 24 & CEP110 & 2 \\
10 & GLI3 & 4 & 25 & HS.541237 & 2 \\
11 & CDKN2AIP & 4 & 26 & KLF6 & 2 \\
12 & CREG1 & 3 & 27 & PRKCB1-2 & 2 \\
13 & FOXE1-2 & 3 & 28 & MAK & 2 \\
14 & CSNK1A1 & 3 & 29 & CCL16 & 2 \\
15 & TUB & 3 & 30 & IL23A & 2 \\
\hline
\end{tabular}

Table 4 The performance of the IBCGA and sequential backward selection (SBS) methods

\begin{tabular}{lllll}
\hline Method & $\boldsymbol{w}=\mathbf{0 . 8}$ & $\boldsymbol{w}=\mathbf{0 . 8}$ & $\boldsymbol{w}=\mathbf{1 . 0}$ & $\boldsymbol{w}=\mathbf{1 . 0}$ \\
& Acc (\%) & Asurv (\%) & Acc (\%) & Asurv (\%) \\
\hline IBCGA $\left(S_{a}\right)$ & 95.95 & 70.01 & 97.43 & 69.21 \\
IBCGA $\left(S_{b}\right)$ & 92.02 & 63.81 & 94.73 & 66.91 \\
SBS & 93.59 & 74.81 & 91.03 & 70.92 \\
\hline
\end{tabular}

free survival area. Therefore, Table 5 presents the 17 transcripts of the 16 genes obtained using the SBS method with $w=0.8$. The 16 genes regarded as a candidate set of biomarkers (the 16-gene set) can be further analyzed.

\section{Analyzing identified 16-gene set}

To investigate the abilities of individual genes to predict distant metastasis, the rankings by classification ability, disease-free survival area, and p-value using the training dataset are analyzed. The classification ability ranking is derived according to the classification accuracy using a single gene and the SVM classifier with a parameter setting using a grid search method. Table 5 lists the rankings of classification ability, disease-free survival area,
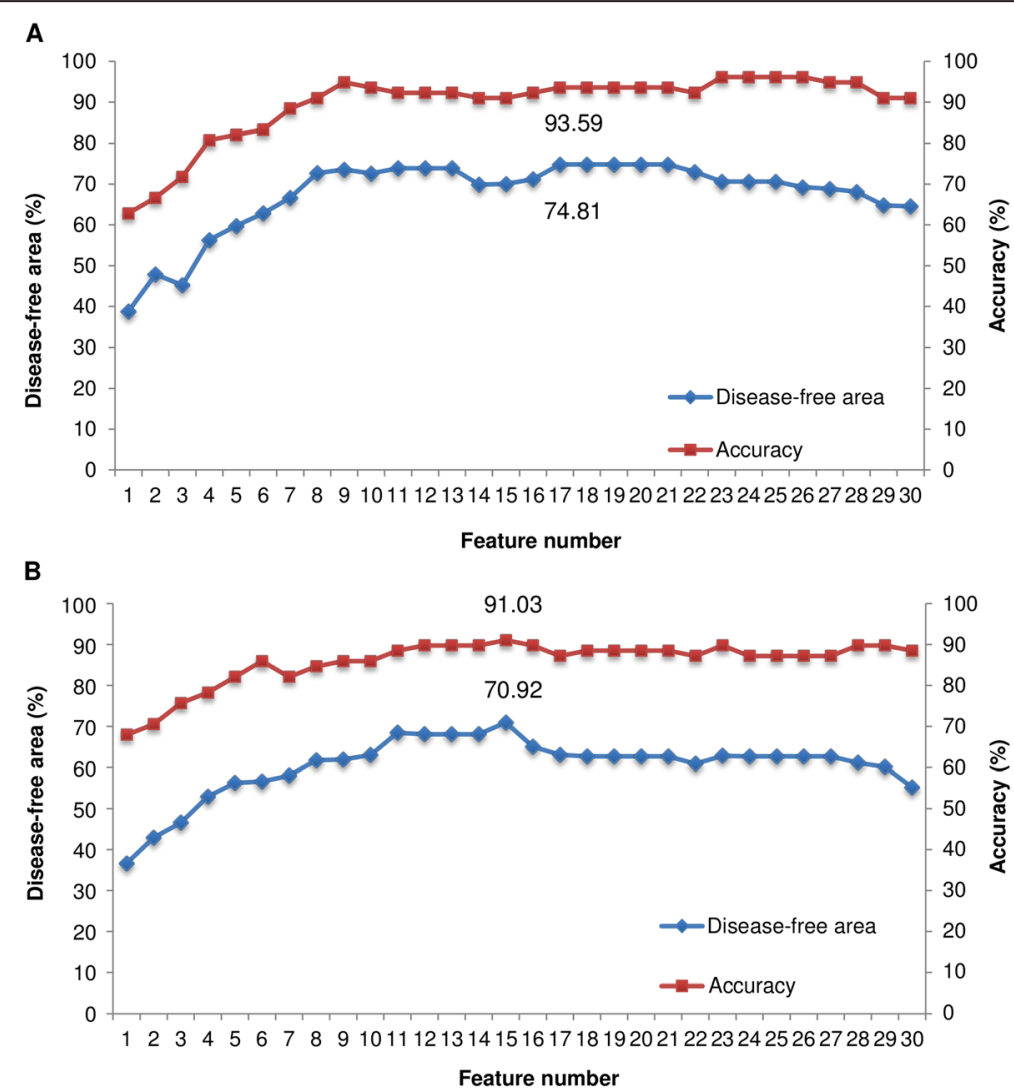

Figure 2 The prediction accuracy and disease-free survival area obtained using the sequence backward selection method. (A) $W=0.8$ (B) $w=1.0$ 


\begin{tabular}{|c|c|c|c|}
\hline Transcript & $\begin{array}{l}\text { Classification } \\
\text { ability\% (Rank) }\end{array}$ & $\begin{array}{l}\text { Disease-free survival } \\
\text { area\% (Rank) }\end{array}$ & $\begin{array}{l}\text { Rank } \\
\text { (p-value) }\end{array}$ \\
\hline$\overline{C C L 16}$ & $74.36(1)$ & $43.58(2)$ & 5 \\
\hline GLI3 & 71.79 (2) & $34.43(6)$ & 15 \\
\hline TUB & 71.79 (3) & 35.71 (4) & 13 \\
\hline PRKCB1-1 & $70.51(4)$ & $34.51(5)$ & 3 \\
\hline ZAP70 & 67.95 & $29.53(8)$ & 14 \\
\hline ELF5 & $67.95(6)$ & $26.70(10)$ & 17 \\
\hline EDN1 & $66.67(7)$ & 31.09 (7) & 9 \\
\hline SF1 & $66.67(8)$ & $25.87(11)$ & 8 \\
\hline CREG1 & $66.67(9)$ & $23.40(14)$ & 2 \\
\hline MAST1 & $65.38(10)$ & $23.71(13)$ & 12 \\
\hline CSNK1A1 & $64.10(11)$ & $24.95(12)$ & 11 \\
\hline HDAC9 & $64.10(12)$ & $27.78(9)$ & 10 \\
\hline MAK & $62.82(13)$ & $38.76(3)$ & 4 \\
\hline CCL15 & $61.54(14)$ & $22.94(15)$ & 6 \\
\hline PRKCB1-2 & $61.54(15)$ & $16.73(17)$ & 1 \\
\hline CDKN2AIP & $61.54(16)$ & $46.00(1)$ & 16 \\
\hline FOXE1-1 & $60.26(17)$ & $19.60(16)$ & 7 \\
\hline
\end{tabular}

and p-value for every transcript in the 16-gene set. Table 5 reveals that the distributions of rankings for the three metrics are diverse. All of the p-values of the 17 transcripts are very small so a comparison of $\mathrm{p}$-value ranking is not meaningful. The Person correlation coefficient between the ranks based on the two metrics (accuracy and survival area) is not very high $(R=0.4755)$. The gene chemokine (C-C motif) ligand 16 (CCL16) provides the highest classification accuracy (74.36\%) and the second largest area of disease-free survival (43.58\%). The gene CDKN2A-interacting protein (CDKN2AIP) has a disease-free survival area of $46.00 \%$ (rank 1) and a classification accuracy of $61.54 \%$ (rank 16). The significant variation among the rankings of genes based on the three metrics is discussed below.

The effective discrimination between distant and nondistant metastases can be achieved using a set of interacted genes that are involved in various signaling pathways, rather than a set of mutually independent genes. The LOOCV accuracy of $93.59 \%$ that is obtained using the 16-gene set substantially exceeds that obtained using a single gene. On the other hand, when all of the 17 transcripts are used to predict distant metastasis, the disease-free survival area (Asurv) is $74.81 \%$, which is very close to the real area of $73.21 \%$. Moreover, this area $(74.81 \%)$ is much larger than that obtained using a single gene. The expression level of individual genes using the microarray technique cannot be used reliably to discriminate between samples of distant and non-distant metastases. Several factors determine patient survival, including gene expression and the nature of the therapy. Therefore, survival curve provides valuable information, but care must be taken in using the survival curves of individual genes. In brief, the rankings of individual genes for the three metrics based on univariate analysis can be used in initial screening in coarse-to-fine gene selection. In this work, the discovery of biomarkers in the fine stage takes into account a set of genes by combining both the classification ability and disease-free survival area.

Numerous prediction methods discover biomarkers by searching for a set of genes that can provide highly accurate performance of LOOCV or $10-C V[19,20]$. Many sets may comprise a small number of genes that can achieve the same goal. The highest LOOCV accuracy that can be achieved using SVM with ten genes without considering the disease-free survival curves is $97.43 \%$ $(w=1.0$, Table 4$)$. The proposed method of applying SBS to a combination of promising gene sets aims to identify a set of reliable biomarkers for predicting distant metastasis. Notably, some genes in the set of 30 transcripts (Table 3) but not in the identified 16-gene set may also be potential biomarkers.

\section{Evaluation of biomarkers using an independent cohort}

To evaluate generalizability of the identified gene sets, an independent cohort of 26 samples was utilized. Table 6 shows the performance of the IBCGA and the SBS methods by performing 30 runs on the 26 test samples. The training accuracies for $w=1.0$ and 0.8 are $88.80 \%$ and $88.88 \%$, respectively, which are very close to each other. However, the test accuracies for $w=1.0$ and 0.8 are $50.41 \%$ and $61.08 \%$, respectively. The test accuracy obtained using disease-free survival curves $(w=0.8)$ is larger than that obtained using no survival curve $(w=$ $1.0)$ that is improved by $10.67 \%$. The test accuracies of the SBS method for $w=1.0$ and 0.8 are $53.84 \%$ and $65.38 \%$, respectively. The improvement in the test accuracy is $11.54 \%$. The improvement in the mean test accuracy using the disease-free survival curves is $11.10 \%$. The results also reveal that the SBS method that includes the IBCGA outperforms the IBCGA method alone for both $w=1.0$ and 0.8 .

The SBS method with $w=0.8$ is the best method, yielding an LOOCV accuracy of $93.59 \%$ and a predicted disease-free survival area of $74.81 \%$, which is very close to the real disease-free survival area of $73.21 \%$. The accuracy of the independent test using an SVM classifier with 17 transcripts is $65.38 \%$. The SVM ensemble of 30 SVM classifiers of which each uses five transcripts that are randomly selected from the 17 transcripts can yield the test accuracy of $76.92 \%$. The intractable problem of overtraining has been alleviated by the additional use of survival curves in the proposed optimization method. 
Table 6 The test accuracies of the IBCGA and sequence backward selection (SBS) methods from 30 runs

\begin{tabular}{llll}
\hline Method & No. of features & Training Acc (\%) & Test Acc (\%) \\
\hline IBCGA $(w=1.0)$ & 10 & $88.80 \pm 3.92$ & $50.41 \pm 6.56$ \\
IBCGA $(w=0.8)$ & 10 & $88.88 \pm 3.27$ & $61.08 \pm 7.35$ \\
SBS $(w=1.0)$ & 15 & 91.03 & $53.84(14 / 26)$ \\
SBS $(w=0.8)$ & 17 & 93.59 & $65.38(17 / 26)$ \\
SVM ensemble & 17 & 93.59 & $76.92(20 / 26)$ \\
\hline
\end{tabular}

However, this performance should be not sufficient to select a putative set of biomarkers. Increase of the number of samples can further mitigate the overtraining problem.

\section{Performance comparison of various gene sets}

Table 7 shows the type of regulator and location of its protein product, as well as the related cancer genes in the 16-gene set. The 16 genes are as follows: 1) endothelin 1 (EDN1), 2) casein kinase 1, alpha 1 (CSNK1A1), 3)

Table 7 The type of regulator and location of its protein product, as well as the related cancer of the genes in the 16-gene set

\begin{tabular}{|c|c|c|c|c|}
\hline No & $\begin{array}{l}\text { Gene } \\
\text { name }\end{array}$ & $\begin{array}{l}\text { Type of } \\
\text { regulator }\end{array}$ & Location & Related cancer \\
\hline 1 & EDN1 & Cytokine & $\begin{array}{l}\text { Extracellular } \\
\text { space }\end{array}$ & $\begin{array}{l}\text { Lung cancer } \\
\text { breast cancer }\end{array}$ \\
\hline 2 & CSNK1A1 & Kinase & Cytoplasm & NSCLC \\
\hline 3 & CCL15 & Cytokine & $\begin{array}{l}\text { Extracellular } \\
\text { space }\end{array}$ & NSCLC \\
\hline 4 & SF1 & $\begin{array}{l}\text { Transcription } \\
\text { regulator }\end{array}$ & Nucleus & Lung cancer \\
\hline 5 & PRKCB1 & Kinase & Cytoplasm & Lung cancer \\
\hline 6 & MAST1 & Kinase & Cytoplasm & Breast cancer \\
\hline 7 & ZAP70 & Kinase & $\begin{array}{l}\text { Plasma } \\
\text { membrane }\end{array}$ & Colorectal cancer \\
\hline 8 & CCL16 & Cytokine & $\begin{array}{l}\text { Extracellular } \\
\text { space }\end{array}$ & $\begin{array}{l}\text { Mammary } \\
\text { Adenocarcinoma }\end{array}$ \\
\hline 9 & ELF5 & $\begin{array}{l}\text { Transcription } \\
\text { regulator }\end{array}$ & Nucleus & Cancer \\
\hline 10 & CDKN2AIP & $\begin{array}{l}\text { Transcription } \\
\text { regulator }\end{array}$ & Nucleus & Cancer \\
\hline 11 & HDAC9 & $\begin{array}{l}\text { Transcription } \\
\text { regulator }\end{array}$ & Nucleus & Medulloblastoma \\
\hline 12 & GLI3 & $\begin{array}{l}\text { Transcription } \\
\text { regulator }\end{array}$ & Nucleus & Cancer \\
\hline 13 & FOXE1 & $\begin{array}{l}\text { Transcription } \\
\text { regulator }\end{array}$ & Nucleus & Thyroid \\
\hline 14 & MAK & Kinase & Cytoplasm & Prostate cancer \\
\hline 15 & $T U B$ & $\begin{array}{l}\text { Transcription } \\
\text { regulator }\end{array}$ & Cytoplasm & $\begin{array}{l}\text { Relation to ocular } \\
\text { diseases }\end{array}$ \\
\hline 16 & CREG1 & $\begin{array}{l}\text { Transcription } \\
\text { regulator }\end{array}$ & Nucleus & $\begin{array}{l}\text { Inhibitor of } \\
\text { apoptosis }\end{array}$ \\
\hline
\end{tabular}

chemokine (C-C motif) ligand 15 (CCL15), 4) splicing factor $1(S F 1), 5)$ gene protein kinase $\mathrm{C}$ beta (PRKCB1), 6) microtubule-associated serine/threonine protein kinase 1 (MAST1), 7) zeta-chain associated protein kinase $70 \mathrm{kDa}(Z A P 70), 8)$ chemokine (C-C motif) ligand 16 (CCL16), 9) E74-like factor 5 (ELF5), 10) CDKN2Ainteracting protein $(C D K N 2 A I P), 11)$ histone deacetylase 9 (HDAC9), 12) GLI family zinc finger 3 (GLI3), 13) forkhead box E1 (FOXE1), 14) male germ cell-associated kinase $(M A K), 15)$ tubby protein homolog $(T U B)$, and 16) cellular repressor of E1A-stimulated genes 1 (CREG1). The 16-gene set can be categorized into two subsets. One subset has five known lung-cancer-related genes (named 5-gene set), including EDN1, CSNK1A1, CCL15, SF1 and PRKCB1. The other subset (11-gene set) consists of nine cancer-related genes and two potential biomarkers (TUB and CREG1).

A number of lung cancer-related genes in the literature are considered for further comparison and analysis. Since EMT is known to be involved in tumor malignancy, some mesenchymal-related genes of gastric cancer, including WNT5A, CDH2, PDGFRB, EDNRA, $R O B O 1, R O R 2$, and $M E F 2 C$ that are activated by an EMT regulator, are also examined [28]. Table 8 presents the 32 genes and their relevant papers [28-39]. The same sequence backward selection (SBS) method is applied to the 32-gene set, yielding a set of nine genes that can be used to accurately predict distant metastasis of lung cancer. The 9-gene set consists of MEF2C, MMP-2, ID2, CDH2, WNT5A, CDH1, TGFB1, $M M P-9$, and TWIST2. Notably, cigarette smoke induces WNT5A-coupled PKC activity during lung carcinogenesis, which causes Akt activity and anti-apoptosis in lung

Table 8 The 32 lung cancer-related genes and their relevant papers

\begin{tabular}{lll}
\hline No. & Gene name & Paper \\
\hline 1 & CDH2, PDGFRB, ROBO1, ROR2, & Ohta et al. [28] \\
& MEF2C, WNT5A, EDNRA & \\
2 & ZEB1, ZEB2, CDS1, ST14, FGFR1, & Gemmill et al. [29] \\
& TWIST1, TWIST2, TGFB1 & \\
3 & VIM, MUC1, S100A4, FOXQ1 & Feng et al. [30] \\
4 & ID2, TGFB1 & Yoshikawa et al. [31] \\
5 & SNAI1, MMP-9, MMP-7, MMP-2, & Ward et al. [32] \\
6 & SIOOA4, TGFB1 & \\
7 & HMGA2, TTF1 & Qi et al. [33] \\
8 & SYK & Singh et al. [34] \\
9 & TYRO3, AXL, PDGFRB & Thomson et al. [35] \\
10 & SIP1, ZEB1 & Takeyama et al. [36] \\
11 & TCF4 & Xiang et al. [37] \\
12 & SNAI1, TGFB1 & Matsuno et al. [38] \\
\hline
\end{tabular}


cancer [40]. The two mesenchymal-related genes, $\mathrm{CDH} 2$ and $M E F 2 C$ were also selected into the 9-gene set.

Table 9 presents performance comparisons of various gene sets in terms of the prediction accuracy. The training accuracy, disease-free survival area, and independent test accuracy obtained using the 32-gene set are $65.38 \%$, $34.47 \%$, and $50.00 \%$, respectively. The performance of the 32-gene set is not good, especially for the independent test. This lung cancer-related gene set is not designed especially to predict distant metastasis of lung cancer. The 9-gene set yields a training accuracy of $73.07 \%$, a disease-free survival area of $55.70 \%$, and an independent test accuracy of $80.76 \%$. As a result, the 9gene set that is obtained using the SBS method is more effective in identifying the distant metastasis than is the 32-gene set, and is worthy of further validation.

The 16-gene set for predicting distant metastasis is identified using microarray and survival data at the same time. The test accuracy is $76.92 \%$ for the 16 -gene set using an SVM ensemble classifier. To analyze the 16gene set further, its two subsets (5- and 11-gene sets) are independently evaluated using the same prediction method as that used to evaluate the 16-gene set, and the results are shown in Table 9. The training accuracy, disease-free survival area, and independent test accuracy achieved using the 5-gene set with six transcripts are $78.25 \%, 52.33 \%$, and $76.92 \%$, respectively, which are close to those achieved using the 9-gene set. The 11gene set of EMT and cancer-related genes has a higher training accuracy of $87.18 \%$, which is nearly equal to the survival area of $53.37 \%$, and has a smaller test accuracy of $69.23 \%$ than the 5 -gene set. To compare individual genes in terms of their prediction performance, three representative genes in the 16-gene set were selected. The top genes with the highest training accuracy and

Table 9 Performance comparison among various gene sets

\begin{tabular}{llll}
\hline Gene set & $\begin{array}{l}\text { Training } \\
\text { Acc (\%) }\end{array}$ & $\begin{array}{l}\text { Training } \\
\text { Asurv (\%) }\end{array}$ & Test Acc (\%) \\
\hline $\begin{array}{l}\text { 32-gene (lung cancer } \\
\text { related) }\end{array}$ & 65.38 & 34.47 & $50.00(13 / 26)$ \\
$\begin{array}{l}\text { 9-gene (SBS from } \\
\text { the 32-gene set) }\end{array}$ & 73.07 & 55.70 & $80.76(21 / 26)$ \\
$\begin{array}{l}\text { 16-gene (EMT related, } \\
\text { SVM ensemble) }\end{array}$ & 93.59 & 74.81 & $76.92(20 / 26)$ \\
$\begin{array}{l}\text { 11-gene (EMT and } \\
\text { cancer related) }\end{array}$ & 87.18 & 53.37 & $69.23(18 / 26)$ \\
$\begin{array}{l}\text { 5-gene (EMT and lung } \\
\text { cancer related) }\end{array}$ & 78.25 & 52.33 & $76.92(20 / 26)$ \\
$\begin{array}{l}\text { 1-gene (CCL16, } \\
\text { maximum ACC) }\end{array}$ & 74.36 & 43.58 & $57.69(15 / 26)$ \\
$\begin{array}{l}\text { 1-gene (CDKN2AIP, } \\
\text { maximum Asurv) }\end{array}$ & 61.54 & 46.00 & $76.92(20 / 26)$ \\
\hline
\end{tabular}

The value of Asurv is $73.21 \%$ for real classes of samples in the training dataset. disease-free survival area are CCL16 (74.36\%) and CDKN2AIP (46.00\%), respectively. Figure 3 plots the Kaplan-Meier survival curves and their corresponding disease-free survival areas for real and predicted classes obtained using various gene sets.

The large disease-free survival area (74.81\%) of predicted classes, obtained using the 16-gene set, is remarkably very close to that of the real classes $(73.21 \%)$. To investigate the ability of this set to predict patient's disease-free survival time, the support vector regression in the LIBSVM [41] is used to establish a survival prediction model. Figure 4 shows that, in the estimate of the disease-free survival time, the correlation coefficient between the real and predicted disease-free survival times is $R=0.9672$. The mean survival prediction error is 3.99 months. This result reveals that the 16-gene set is also effective in predicting disease-free survival times of patients.

\section{Examination of the 16 putative biomarkers}

The 16 putative biomarkers herein are examined with reference to the relevant papers. Five of the 16 genes have been shown to be related to lung cancer progression. Decreased expression of EDN1 has been reported in primary lung cancers, possibly owing to the high methylation in the $\mathrm{CpG}$ island of its intron 1 and exon 2 junction [42]. Overexpressions of EDN1 and EDNRA were already reported as being associated with impaired survival in human breast cancer [43]. Expression analysis reveals that hypoxia-induced lung cancer relatedbiomarkers $H I F$ and its modulating proteins, including CSNK1A1, are significantly down-regulated [44]. The CCL15 level correlates with response to combination therapy with erlotinib and celecoxib in patients with NSCLC [45]. Chemokine CCL15 is the most significant marker that is associated with increased odds of short survival [46]. Splicing factor SF1 participates in the ATP-dependent formation of the spliceosome complex [47]. Down-regulation of the oncogenic serine/argininerich splicing factor 1 (SF1) leads to the skipping of an exon that is overexpressed in primary lung tumors [48]. PRKCB belongs to a family of serine/threonine-specific kinases and is predominantly activated by diacylglycerol, calcium, and phorbol ester. The two splice-variants are called PRKCB1 and PRKCB2. PRKCB1 exists in lung cancer cell lines in the context of enzastaurin-induced proliferation and kinase inhibition, determined by using exon sequencing, immunoblotting, and cytotoxicity assays in NSCLC and SCLC cell lines [49].

The nine cancer-related genes are briefly described below. Breast cancer cell lines that harbor Notch gene rearrangements are uniquely sensitive to the inhibition of Notch signaling, and the overexpression of MAST1 or $M A S T 2$ gene fusions has a proliferative effect [50]. The 

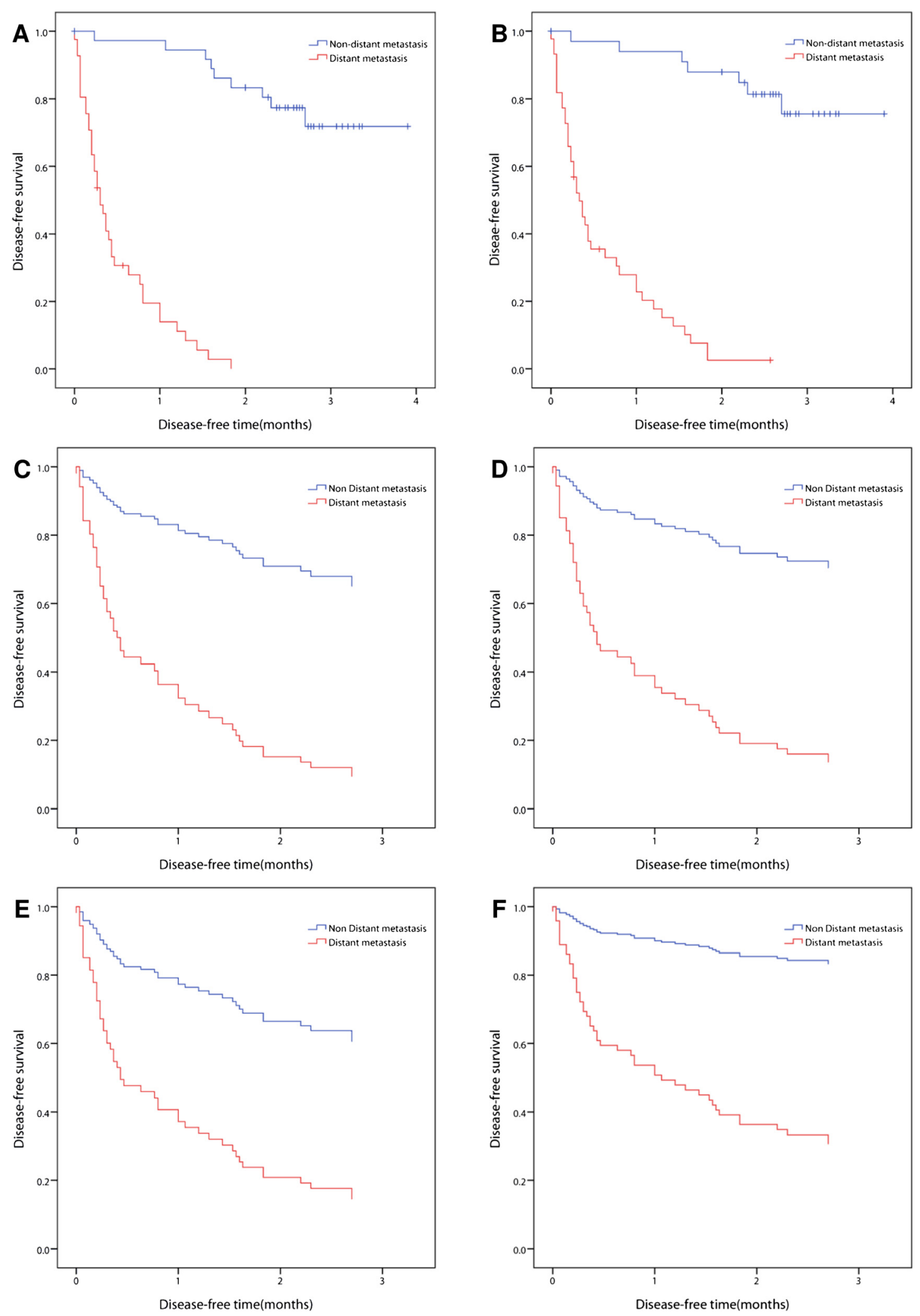

Figure 3 The disease-free survival areas of various gene sets using Kaplan-Meier survival curves. There are 37 non-distant- (in blue) and 41 distant-metastasis samples (in red). (A) real class (73.21\%), (B) 16-gene set (74.81\%), (C) 11-gene set (53.37\%), (D) 5-gene set (52.33\%), (E) CCL16 (43.58\%) and (F) CDKN2AIP (46.00\%). 


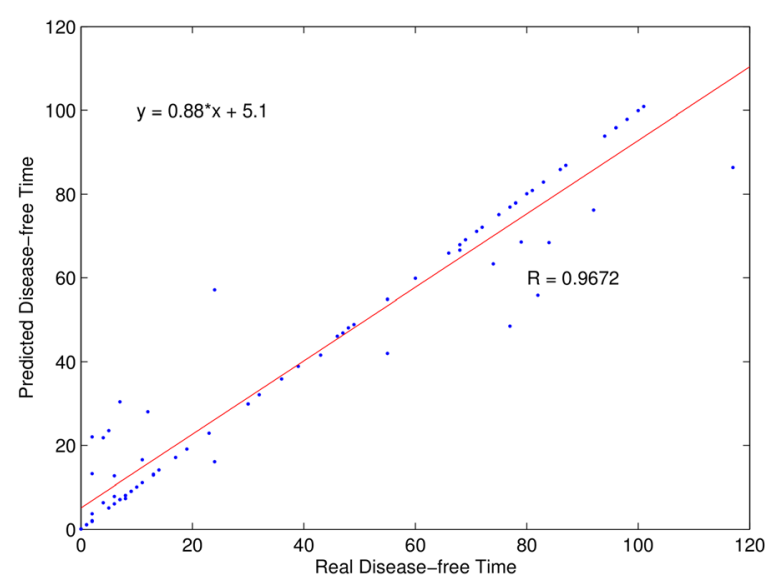

Figure 4 The predicted disease-free survival time using the support vector regression with the 16-gene set.

overexpressed gene ZAP70 significantly relates to prognostic factors such as tumor size, advanced stage, invasive depth, lymph node metastasis and differentiation [51]. An adenovirus that encodes CCL16, when injected into established nodules significantly delayed tumor growth [52]. ELF5 can transactivate through the sequences of ETS transcription factors. ELF5 is localized to human chromosome $11 \mathrm{p} 13-15$, which is a region that frequently undergoes loss of heterozygosity in several types of carcinoma, including those of the breast, kidney and prostate [53]. $C D K N 2 A I P$ has a known role in tumorigenesis [54]. A functional role of HDAC5 and HDAC9 in tumor cell growth in medulloblastoma cell lines has been reported [55]. Three sonic hedgehog effectors, GLI1, GLI2, and GLI3, regulate the transcription of diverse genes that are involved in cell growth and cell proliferation [56]. FOXE1 (TTF-2) is a thyroid-specific transcription factor and a marker in thyroid tumors. The lung is the most common distant metastatic site for thyroid carcinomas [57]. $M A K$ is a direct transcriptional target of androgen receptor which plays an important role in the normal development of prostate as well as in the progression of prostate cancer [58]. Phylogenetically, TULP3 is the family member that is most closely related to TUB. TULP3 is detected at high levels in human RNA from testes, ovaries, the thyroid, and the spinal chord [59]. TUB encodes a protein of 561 amino acids that is highly expressed in a number of tissues examined, including the heart, brain, ovary, thyroid, spinal chord, and retina and it maps to chromosome 11p15.4 [60]. The overexpression of CREG1 reduces cell proliferation in immortal LFS and cancer cell lines. CREG1 has been identified as a potent inhibitor of apoptosis [61]. The cooperation of CREG1 and p16 (INK4a) inhibits the expression of cyclin A and cyclin B by inhibiting promoter activity, reducing mRNA and protein levels, and these proteins are required for S-phase entry and G2/M transition $[61,62]$.

After these putative biomarkers are mapped into the KEGG pathway database (http://www.genome.jp/kegg/), several signaling pathways that involve lung cancer metastasis are identified. Mitogen-activated protein kinase signaling pathway, NF-kappa B signaling pathway, and immune response IL-1 signaling pathway have been reported in lung cancer metastasis. While regulating proliferation, gene expression, differentiation, mitosis, cell survival, and apoptosis, the mitogen-activated protein kinase signaling pathway has long been viewed as an attractive pathway for anticancer therapies [63]. A higher nuclear factor-kappa B expression pattern is associated with more advanced stages of oncogenesis and it will expand related pathways for invasion and metastasis in lung cancer [64]. Abnormal IL-1 expression and its related pathway seem to be related to IKK alpha/beta activation, p65 translocation and transcription activity, and associated to migration of cancer cells [65]. We suggest that the pathways that are associated with these biomarkers might play an important role in NSCLC metastasis. This inference offers an opportunity to expand greatly our knowledge of the expression patterns in NSCLC. Considering together the expression status of biomarkers, related signaling pathways, and clinical outcome will further reveal the roles of the biomarkers in lung cancer metastasis. An enhanced understanding of the basic biological mechanisms of NSCLC will likely facilitate development of improved methods for survival prediction.

\section{Conclusions}

This work proposes a novel methodological approach to discovering a set of prognostic biomarkers for predicting the distant metastasis of lung cancer through simultaneous utilization of microarray and survival data. The presented optimization method uses an objective function to maximize both prediction accuracy and the disease-free survival area to identify a set of biomarkers. The additional use of clinical disease-free survival time can greatly facilitate the discovery of biomarkers and the prediction of survival time. The proposed method that combines both microarray and survival data can also alleviate the problem of overtraining that arises from the insufficiency of samples. The experimental results herein show that a combination of multiple biomarkers may increase diagnostic sensitivity and specificity over those obtained using individual biomarkers. The proposed method has high generalizability in the discovery of prognostic biomarkers not only for the distant metastasis of lung cancer, but also for other cancers using microarray data and clinical outcomes. 
Few studies have investigated the discovery of prognostic biomarkers for predicting the distant metastasis of lung cancer. This work identified a set of 16 prognostic biomarkers that can be used to predict distant metastasis with high accuracies, a leave-one-out crossvalidation accuracy of $93.59 \%$, an independent test accuracy of $76.92 \%$, a large predicted disease-free survival area of $74.81 \%$ (close to $73.21 \%$ for real survival area), and a mean survival prediction error of 3.99 months. Closely examining the 16-gene set by mapping these genes into the KEGG pathway database reveals some signaling pathways that are involved in lung cancer metastasis. Future work will incorporate these findings of signaling pathways with related biomarkers and clinical outcomes to develop novel methods for predicting distant metastasis and the survival times of patients with early-stage NSCLC.

\section{Additional files}

Additional file 1: Table S1. The data set of 78 lung cancer patients for training.

Additional file 2: Table S2. The data set of 26 lung cancer patients for test.

\section{Competing interests}

The authors declare that they have no competing interests.

\begin{abstract}
Authors' contributions
HLH carried out the system design, participated in the analysis and discussion of the study, and drafted the manuscript. YCW carried out the data collection, participated in the design and discussion of biomarker discovery. LJS participated in the analysis of biomarkers and their pathways, and helped to draft the manuscript. YJH participated in the survey of related work, the experimental analysis, and manuscript preparation. PC carried out the program implementation, and participated in the computational analysis. WLC, HCL and WCC participated in the statistical analysis and examination of biomarkers. SYH conceived of the study, and participated in its design and coordination, and helped to draft the manuscript. All authors read and approved the final manuscript.
\end{abstract}

\section{Acknowledgements}

This work was funded by National Science Council of Taiwan under the contract number NSC-103-2221-E-009-117-, and "Center for Bioinformatics Research of Aiming for the Top University Program" of the National Chiao Tung University and Ministry of Education, Taiwan, R.O.C. for the project 103 W962. This work was also supported in part by the UST-UCSD International Center of Excellence in Advanced Bioengineering sponsored by the Taiwan National Science Council I-RiCE Program under Grant Number: NSC-1032911-I-009-101-. This work was also supported by Taipei Veterans General Hospital in Taiwan with project VGHIRB No. 2013-04-015 AC.

\footnotetext{
Author details

${ }^{1}$ Institute of Bioinformatics and Systems Biology, National Chiao Tung University, Hsinchu, Taiwan. ${ }^{2}$ Department of Biological Science and Technology, National Chiao Tung University, Hsinchu, Taiwan. ${ }^{3}$ Division of Thoracic Surgery, Department of Surgery, Taipei Veterans General Hospital, Taipei, Taiwan. ${ }^{4}$ Institute of Systems Biology and Bioinformatics, National Central University, Taoyuan, Taiwan. ${ }^{5}$ Institute of Molecular Medicine and Bioengineering, National Chiao Tung University, Hsinchu, Taiwan.

${ }^{6}$ Department of Computer Science, Tunghai University, Taichung, Taiwan.
}

Received: 10 June 2014 Accepted: 13 January 2015

Published online: 21 February 2015

\section{References}

1. Yang P. Epidemiology of lung cancer prognosis: quantity and quality of life. Methods Mol Biol. 2009;471:469-86.

2. Kulasingam V, Diamandis EP. Strategies for discovering novel cancer biomarkers through utilization of emerging technologies. Nat Clin Pract Oncol. 2008;5(10):588-99.

3. Thiery JP. Epithelial-mesenchymal transitions in tumour progression. Nat Rev Cancer. 2002;2(6):442-54

4. Lyons JG, Lobo E, Martorana AM, Myerscough MR. Clonal diversity in carcinomas: its implications for tumour progression and the contribution made to it by epithelial-mesenchymal transitions. Clin Exp Metastasis. 2008;25(6):665-77.

5. Lee JM, Dedhar S, Kalluri R, Thompson EW. The epithelial-mesenchymal transition: new insights in signaling, development, and disease. J Cell Biol. 2006;172(7):973-81.

6. Walser T, Cui X, Yanagawa J, Lee JM, Heinrich E, Lee G, et al. Smoking and lung cancer: the role of inflammation. Proc Am Thorac Soc. 2008;5(8):811-5.

7. Chow G, Tauler J, Mulshine JL. Cytokines and growth factors stimulate hyaluronan production: role of hyaluronan in epithelial to mesenchymal-like transition in non-small cell lung cancer. J Biomed Biotechnol. 2010;2010:485468.

8. Shimamura T, Imoto S, Shimada Y, Hosono Y, Niida A, Nagasaki M, et al. A novel network profiling analysis reveals system changes in epithelial-mesenchymal transition. Plos One. 2011;6(6):e20804.

9. Shih JY, Yang PC. The EMT regulator slug and lung carcinogenesis. Carcinogenesis. 2011;32(9):1299-304.

10. Kawata M, Koinuma D, Ogami T, Umezawa K, Iwata C, Watabe T, et al. TGF-beta-induced epithelial-mesenchymal transition of A549 lung adenocarcinoma cells is enhanced by pro-inflammatory cytokines derived from RAW 264.7 macrophage cells. J Biochem. 2012;151(2):205-16.

11. Thomson S, Petti F, Sujka-Kwok I, Epstein D, Haley JD. Kinase switching in mesenchymal-like non-small cell lung cancer lines contributes to EGFR inhibitor resistance through pathway redundancy. Clin Exp Metastasis. 2008;25(8):843-54

12. Fuchs BC, Fujii T, Dorfman JD, Goodwin JM, Zhu AX, Lanuti M, et al. Epithelial-to-mesenchymal transition and integrin-linked kinase mediate sensitivity to epidermal growth factor receptor inhibition in human hepatoma cells. Cancer Res. 2008;68(7):2391-9.

13. Sun Z, Yang P. Gene expression profiling on lung cancer outcome prediction: present clinical value and future premise. Cancer Epidemiol Biomarkers Prev. 2006;15(11):2063-8.

14. Chen HY, Yu SL, Chen CH, Chang GC, Chen CY, Yuan A, et al. A five-gene signature and clinical outcome in non-small-cell lung cancer. N Engl J Med. 2007;356(1):11-20.

15. Lau SK, Boutros PC, Pintilie M, Blackhall FH, Zhu CQ, Strumpf D, et al. Three-gene prognostic classifier for early-stage non small-cell lung cancer. J Clin Oncol. 2007;25(35):5562-9.

16. Dudoit S, Fridlyand J, Speed TP. Comparison of discrimination methods for the classification of tumors using gene expression data. J Am Stat Assoc. 2002;97(457):77-87.

17. Eisen MB, Spellman PT, Brown PO, Botstein D. Cluster analysis and display of genome-wide expression patterns. Proc Natl Acad Sci U S A. 1998;95(25):14863-8.

18. Liu HY, Kho AT, Kohane IS, Sun Y. Predicting survival within the lung cancer histopathological hierarchy using a multi-scale genomic model of development. Plos Med. 2006;3(7):1090-102.

19. Huang HL, Chang FL. ESVM: evolutionary support vector machine for automatic feature selection and classification of microarray data. Bio Systems. 2007;90(2):516-28.

20. Huang HL, Lee CC, Ho SY. Selecting a minimal number of relevant genes from microarray data to design accurate tissue classifiers. Bio Systems. 2007;90(1):78-86.

21. Ho SY, Shu LS, Chen JH. Intelligent evolutionary algorithms for large parameter optimization problems. leee Trans Evol Comput. 2004;8(6):522-41.

22. Shi W, Banerjee A, Ritchie ME, Gerondakis S, Smyth GK. Illumina WG-6 BeadChip strips should be normalized separately. Bmc Bioinformatics. 2009;10:372.

23. Sugiura H, Yamada K, Sugiura T, Hida T, Mitsudomi T. Predictors of survival in patients with bone metastasis of lung cancer. Clin Orthop Relat Res. 2008;466(3):729-36. 
24. Chawla NV, Bowyer KW, Hall LO, Kegelmeyer WP. SMOTE: Synthetic minority over-sampling technique. J Artif Intell Res. 2002;16:321-57.

25. Ho SY, Chen JH, Huang MH. Inheritable genetic algorithm for biobjective 0/1 combinatorial optimization problems and its applications. IEEE Trans Syst Man Cybern Part B Cybern Publ IEEE Syst Man Cybern Soc. 2004;34(1):609-20.

26. Tung CW, Ho SY. POPI: predicting immunogenicity of MHC class I binding peptides by mining informative physicochemical properties. Bioinformatics. 2007;23(8):942-9.

27. Huang HL, Lin IC, Liou YF, Tsai CT, Hsu KT, Huang WL, et al. Predicting and analyzing DNA-binding domains using a systematic approach to identifying a set of informative physicochemical and biochemical properties. BMC Bioinformatics. 2011;12 Suppl 1:47.

28. Ohta H, Aoyagi K, Fukaya M, Danjoh I, Ohta A, Isohata N, et al. Cross talk between hedgehog and epithelial-mesenchymal transition pathways in gastric pit cells and in diffuse-type gastric cancers. Br J Cancer. 2009;100(2):389-98.

29. Gemmill RM, Roche J, Potiron VA, Nasarre P, Mitas M, Coldren CD, et al. ZEB1responsive genes in non-small cell lung cancer. Cancer Lett. 2011;300(1):66-78.

30. Feng J, Zhang XS, Zhu HJ, Wang XD, Ni SS, Huang JF. FoxQ1 Overexpression Influences Poor Prognosis in Non-Small Cell Lung Cancer, Associates with the Phenomenon of EMT. Plos One. 2012;7(6):e39937.

31. Yoshikawa M, Hishikawa K, Marumo T, Fujita T. Inhibition of histone deacetylase activity suppresses epithelial-to-mesenchymal transition induced by TGF-beta 1 in human renal epithelial cells. J Am Soc Nephrol. 2007;18(1):58-65.

32. Ward C, Forrest IA, Murphy DM, Johnson GE, Robertson H, Cawston TE, et al. Phenotype of airway epithelial cells suggests epithelial to mesenchymal cell transition in clinically stable lung transplant recipients. Thorax. 2005;60(10):865-71.

33. Qi J, Rice SJ, Salzberg AC, Runkle EA, Liao J, Zander DS, et al. MiR-365 regulates lung cancer and developmental gene thyroid transcription factor 1. Cell Cycle. 2012;11(1):177-86.

34. Singh A, Greninger P, Rhodes D, Koopman L, Violette S, Bardeesy N, et al. A Gene expression signature associated with "K-Ras addiction" reveals regulators of EMT and tumor cell survival. Cancer Cell. 2009;15(6):489-500.

35. Thomson S, Petti F, Sujka-Kwok I, Mercado P, Bean J, Monaghan M, et al. A systems view of epithelial-mesenchymal transition signaling states. Clin Exp Met. 2011;28(2):137-55.

36. Takeyama Y, Sato M, Horio M, Hase T, Yoshida K, Yokoyama T, et al. Knockdown of ZEB1, a master epithelial-to-mesenchymal transition (EMT) gene, suppresses anchorage-independent cell growth of lung cancer cells. Cancer Lett. 2010;296(2):216-24.

37. Xiang $X$, Zhuang $X$, Ju S, Zhang $S$, Jiang $H$, Mu J, et al. miR-155 promotes macroscopic tumor formation yet inhibits tumor dissemination from mammary fat pads to the lung by preventing EMT. Oncogene. 2011;30(31):3440-53.

38. Matsuno Y, Coelho AL, Jarai G, Westvvick J, Hogaboam CM. Notch signaling mediates TGF-beta 1-induced epithelial-mesenchymal transition through the induction of Snail. Int J Biochem Cell Biol. 2012;44(5):776-89.

39. Pallier K, Cessot A, Cote JF, Just PA, Cazes A, Fabre E, et al. TWIST1 a New Determinant of Epithelial to Mesenchymal Transition in EGFR Mutated Lung Adenocarcinoma. Plos One. 2012;7(1):e29954.

40. Whang YM, Jo U, Sung JS, Ju HJ, Kim HK, Park KH, et al. Wnt5a Is Associated with Cigarette Smoke-Related Lung Carcinogenesis via Protein Kinase C. Plos One. 2013;8(1):e53012.

41. Chang C-C, Lin C-J. LIBSVM: a library for support vector machines. ACM Transac Int Syst Technol (TIST). 2011;2(3):27.

42. Takai D, Yagi Y, Wakazono K, Ohishi N, Morita Y, Sugimura T, et al. Silencing of HTR1B and reduced expression of EDN1 in human lung cancers, revealed by methylation-sensitive representational difference analysis. Oncogene. 2001;20(51):7505-13.

43. Wiesmann F, Veeck J, Galm O, Hartmann A, Esteller M, Knuchel R, et al. Frequent loss of endothelin-3 (EDN3) expression due to epigenetic inactivation in human breast cancer. Breast Cancer Research. 2009;11:R34.

44. Srivastava M, Khurana P, Sugadev R. Lung cancer signature biomarkers: tissue specific semantic similarity based clustering of digital differential display (DDD) data. BMC Res Notes. 2012;5:617.

45. Reckamp KL, Gardner BK, Figlin RA, Elashoff D, Krysan K, Dohadwala M, et al. Tumor response to combination celecoxib and erlotinib therapy in non-small cell lung cancer is associated with a low baseline matrix metalloproteinase-9 and a decline in serum-soluble E-cadherin. J Thorac Oncol. 2008;3(2):117-24.
46. Bodelon C, Polley MY, Kemp TJ, Pesatori AC, MCShane LM, Caporaso NE, et al. Circulating levels of immune and inflammatory markers and long versus short survival in early-stage lung cancer. Ann Oncol. 2013;24(8):2073-9.

47. Rino J, Desterro JM, Pacheco TR, Gadella Jr TW, Carmo-Fonseca M. Splicing factors SF1 and U2AF associate in extraspliceosomal complexes. Mol Cell Biol. 2008;28(9):3045-57.

48. de Miguel FJ, Sharma RD, Pajares MJ, Montuenga LM, Rubio A, Pio R. Identification of alternative splicing events regulated by the oncogenic factor SRSF1 in lung cancer. Cancer Res. 2014;74(4):1105-15.

49. Lee SH, Chen TA, Zhou J, Hofmann J, Bepler G. Protein kinase C-beta gene variants, pathway activation, and Enzastaurin activity in lung cancer. Clinical Lung Cancer. 2010;11(3):169-75.

50. Robinson DR, Kalyana-Sundaram S, Wu YM, Shankar S, Cao XH, Ateeq B, et al. Functionally recurrent rearrangements of the MAST kinase and Notch gene families in breast cancer. Nat Med. 2011;17(12):1646-U1163.

51. Huang MY, Wang JY, Chang HJ, Kuo CW, Tok TS, Lin SR. CDC25A, VAV1, TP73, BRCA1 and ZAP70 gene overexpression correlates with radiation response in colorectal cancer. Oncol Rep. 2011;25(5):1297-306.

52. Guiducci C, Di Carlo E, Parenza M, Hitt M, Giovarelli M, Musiani P, et al. Intralesional injection of adenovirus encoding CC chemokine ligand 16 inhibits mammary tumor growth and prevents metastatic-induced death after surgical removal of the treated primary tumor. J Immunol. 2004;172(7):4026-36.

53. Zhou J, Ng AYN, Tymms MJ, Jermiin LS, Seth AK, Thomas RS, et al. A novel transcription factor, ELF5, belongs to the ELF subfamily of ETS genes and maps to human chromosome 11p13-15, a region subject to $\mathrm{LOH}$ and rearrangement in human carcinoma cell lines. Oncogene. 1998;17(21):2719-32.

54. Yi CH, Zheng TZ, Leaderer D, Hoffman A, Zhu Y. Cancer-related transcriptional targets of the circadian gene NPAS2 identified by genome-wide ChIP-on-chip analysis. Cancer Lett. 2009;284(2):149-56.

55. Milde T, Oehme I, Korshunov A, Kopp-Schneider A, Remke M, Northcott P, et al. HDAC5 and HDAC9 in medulloblastoma: novel markers for risk stratification and role in tumor cell growth. Clin Cancer Res. 2010;16(12):3240-52.

56. Krauss S, Foerster J, Schneider R, Schweiger S. Protein phosphatase 2A and rapamycin regulate the nuclear localization and activity of the transcription factor GLI3. Cancer Res. 2008;68(12):4658-65.

57. Nonaka D, Tang Y, Chiriboga L, Rivera M, Ghossein R. Diagnostic utility of thyroid transcription factors Pax8 and TTF-2 (FoxE1) in thyroid epithelial neoplasms. Mod Pathol. 2008:21(2):192-200.

58. Ma AH, Xia L, Desai SJ, Boucher DL, Guan Y, Shih HM, et al. Male germ cell-associated kinase, a male-specific kinase regulated by androgen, is a coactivator of androgen receptor in prostate cancer cells. Cancer Res. 2006;66(17):8439-47.

59. Nishina PM, North MA, Ikeda A, Yan YZ, Naggert JK. Molecular characterization of a novel tubby gene family member, TULP3, in mouse and humans. Genomics. 1998;54(2):215-20.

60. North MA, Naggert JK, Yan YZ, NobenTrauth K, Nishina PM. Molecular characterization of TUB, TULP1, and TULP2, members of the novel tubby gene family and their possible relation to ocular diseases. Proc Natl Acad Sci U S A. 1997;94(7):3128-33.

61. Moolmuang B, Tainsky MA. CREG1 enhances p16(INK4a)-induced cellular senescence. Cell Cycle. 2011;10(3):518-30.

62. Peng F, Han YL, Jie D, Yan CH, Jian K, Bo L, et al. Overexpression of cellular repressor of E1A-stimulated genes inhibits TNF-alpha-induced apoptosis via NF-kappa B in mesenchymal stem cells. Biochem Biophys Res Commun. 2011;406(4):601-7.

63. Sebolt-Leopold JS, Herrera R. Targeting the mitogen-activated protein kinase cascade to treat cancer. Nat Rev Cancer. 2004;4(12):937-47.

64. Batra S, Balamayooran G, Sahoo MK. Nuclear factor-kappaB: a key regulator in health and disease of lungs. Arch Immunol Ther Exp. 2011;59(5):335-51.

65. Cheng CY, Hsieh HL, Sun CC, Lin CC, Luo SF, Yang CM. IL-1 beta induces urokinase-plasminogen activator expression and cell migration through PKC alpha, JNK1/2, and NF-kappaB in A549 cells. J Cell Physiol. 2009;219(1):183-93. 